\title{
Computation of Probability Distribution of Strength of Quasibrittle Structures Failing at Macrocrack Initiation
}

\author{
Jia-Liang Le, M.ASCE${ }^{1}$; Jan Eliáš²; and Zdeněk P. Bažant, Hon.M.ASCE ${ }^{3}$
}

\begin{abstract}
Engineering structures must be designed for an extremely low failure probability, $P_{f}<10^{-6}$. To determine the corresponding structural strength, a mechanics-based probability distribution model is required. Recent studies have shown that quasibrittle structures that fail at the macrocrack initiation from a single representative volume element (RVE) can be statistically modeled as a finite chain of RVEs. It has further been demonstrated that, based on atomistic fracture mechanics and a statistical multiscale transition model, the strength distribution of each RVE can be approximately described by a Gaussian distribution, onto which a Weibull tail is grafted at a point of the probability about $10^{-4}$ to $10^{-3}$. The model implies that the strength distribution of quasibrittle structures depends on the structure size, varying gradually from the Gaussian distribution modified by a far-left Weibull tail applicable for small-size structures, to the Weibull distribution applicable for large-size structures. Compared with the classical Weibull strength distribution, which is limited to perfectly brittle structures, the grafted Weibull-Gaussian distribution of the RVE strength makes the computation of the strength distribution of quasibrittle structures inevitably more complicated. This paper presents two methods to facilitate this computation: (1) for structures with a simple stress field, an approximate closed-form expression for the strength distribution based on the Taylor series expansion of the grafted WeibullGaussian distribution; and (2) for structures with a complex stress field, a random RVE placing method based on the centroidal Voronoi tessellation. Numerical examples including three-point and four-point bend beams, and a two-dimensional analysis of the ill-fated Malpasset dam, show that Method 1 agrees well with Method 2 as well as with the previously proposed nonlocal boundary method. DOI: 10.1061/ (ASCE)EM.1943-7889.0000396. ( ) 2012 American Society of Civil Engineers.
\end{abstract}

CE Database subject headings: Probability distribution; Statistics; Cracking; Size effect; Computation.

Author keywords: Finite weakest link model; Strength statistics; Representative volume element; Structural safety; Fracture; Concrete structures; Composites.

\section{Introduction}

The design of various engineering structures, such as buildings, infrastructure, aircraft, ships, etc., must be targeted at an extremely low failure probability $P_{f}<10^{-6}$ [Nordic Committee for Building Structures (NKB) 1978; Melchers 1987; Duckett 2005]. Since it is impossible to determine the design strength for such a low failure probability by experiment, a physically based model of probability distribution of structural strength is of paramount importance. The type of strength distribution is well understood for structures with two extreme failure behaviors:

1. Perfectly ductile behavior, for which, according to the central limit theorem, the structural strength must have a Gaussian (or normal) cumulative distribution function (cdf) because it is a

\footnotetext{
${ }^{1}$ Assistant Professor, Dept. of Civil Engineering, Univ. of Minnesota, Minneapolis, MN 55455; formerly Graduate Research Assistant, Northwestern Univ., Evanston, IL 60208.

${ }^{2}$ Masaryk-Fulbright Fellow, Northwestern Univ., Evanston, IL 60208; Assistant Professor on leave from Institute of Structural Mechanics, Brno Univ. of Technology, Czech Republic 60100.

${ }^{3}$ McCormick Institute Professor and W. P. Murphy Professor of Civil Engineering and Materials Science, Northwestern Univ., Evanston, IL 60208 (corresponding author). E-mail: z-bazant@northwestern.edu

Note. This manuscript was submitted on July 19, 2011; approved on December 19, 2011; published online on December 23, 2011. Discussion period open until December 1, 2012; separate discussions must be submitted for individual papers. This paper is part of the Journal of Engineering Mechanics, Vol. 138, No. 7, July 1, 2012. CASCE, ISSN 0733-9399/2012/ $7-888-899 / \$ 25.00$.
}

weighted sum of the random strengths of material elements along the failure surface; and

2. Perfectly brittle behavior, for which, according to the extreme value statistics and the weakest-link model for an infinite chain of representative volume elements (RVEs), the structural strength must have a Weibull cdf because the structural failure is triggered by the failure of a single RVE, the size of which is negligible compared with the structure size.

Recent research efforts have been directed to structures made of quasibrittle (i.e., brittle heterogeneous) materials, which include concrete, fiber composites, coarse-grained or toughened ceramics, rocks, sea ice, wood, rigid foams, bones, etc., and most brittle materials at micrometer and submicrometer scales. An important feature of quasibrittle structures is that the size of material inhomogenieties is not negligible compared with the structure size, which causes the behavior of quasibrittle structures to be size dependent. Small-size structures exhibit a quasi-plastic failure behavior whereas large-size structures exhibit a brittle failure behavior. Such a size-dependent failure behavior has by now been well documented by numerous size effect tests (Bažant and Chen 1997; Bažant 2004, 2005).

The present study is limited to a broad class of structures that fail (under controlled load) as soon as a macrocrack initiates from one RVE. The failure corresponds to the maximum load and represents the loss of stability under controlled load, after which the crack propagates dynamically. Such behavior characterizes the structures of positive geometry, defined as structures of a geometry for which the derivative of the stress intensity factor with respect to the crack length at constant load is positive. Statistically, such structure may be modeled as a chain of RVEs, i.e., by the weakest-link model. Therefore, the RVE must be defined as the smallest volume whose 
failure causes the failure of the whole structure (Bažant and Pang 2007). Recently, the random particle model indicated that the RVE size is approximately equal to the autocorrelation length of the spatial variation of material strength (Grassl and Bažant 2009). Therefore, RVE strengths can be assumed to be mutually independent.

Physically, the macrocrack initiation represents the formation of an opened stress-free crack, which occurs at the moment at which the fracture process zone (FPZ) reaches its full size and starts to travel forward, trailed by the stress-free crack. The transition to the opened stress-free crack occurs by localization of microcracks in the FPZ (Bažant and Planas 1998; Eliáš and Bažant 2011; Lin and Labuz 2011). Mechanically, the FPZ can usually be adequately described by the cohesive crack model, although the multidimensional nonlocal damage models can provide a more realistic description. In quasibrittle materials it is next to impossible to determine the location of the tip of the stress-free crack optically, but for practical purposes it is not necessary. The crucial point for adoption of the present study's weakest-link model is that the failure of one RVE triggers the failure of the entire structure.

Bažant and Pang $(2006,2007)$ recently developed a model for the cdf of the strength of one RVE. The model was later refined on the basis of fracture mechanics of nano-cracks propagating by small, activation energy controlled, random jumps through a nano-scale element such as an atomic lattice or a disordered nano-structure (Bažant et al. 2009; Le et al. 2011). Based on the transition rate theory for the fracture of nano-element and a hierarchical seriesparallel coupling model for the statistical multiscale transition, it has been shown that the strength cdf of one RVE can be approximately described as Gaussian distribution whose far left tail is modified by a power law (which is the tail of Weibull distribution).

Because, for quasibrittle structures, the chain underlying the weakest-link model is not infinite but finite, the strength distribution must depend on the structure size and geometry, varying from the Gaussian distribution with a remote Weibull tail for small-size structures, to the Weibull distribution for large-size structures. This finite weakest-link model agrees well with the observed strength histograms of various quasibrittle materials including concrete, fiber composites, industrial ceramics, and dental ceramics (Bažant and Pang 2007; Pang et al. 2008; Bažant et al. 2009; Le and Bažant 2009).

Note that what the joint probability theorem gives is only the structural strength distribution. To predict the failure probability and reliability of actual structures under random loading, the structural strength distribution must be in engineering designs combined (according to the joint-probability theorem) with the load distribution. Because this subject is well understood (e.g., Haldar and Mahadevan 2000), it is not included in this paper.

The weakest-link model with a grafted Gauss-Weibull distribution complicates the computation of the cdf of structural strength. For large enough structures, what matters is only the power-law tail of the cdf of strength of one RVE, which causes the entire cdf of structural strength to follow the Weibull distribution (e.g., Bažant and Pang 2006, 2007; Le et al. 2011). In this case, it is convenient to adopt the concept of equivalent number of RVEs, $N_{e q}$, which represents the number of RVEs that gives the same failure probability for a specimen subjected to uniform uniaxial tension (Bažant and Pang 2007). It can be shown that $N_{e q}$ explicitly depends on the stress field, and it is possible to obtain a closed-form solution for the cdf of strength of large-size structures $\left(N_{e q}>5,000\right)$.

However, for small- and intermediate-size structures, the cdf of structural strength is governed by both the Weibull and Gaussian parts of the grafted distribution of RVE strength, and then $N_{e q}$ depends on both the stress field and the stress magnitude. In this case, a closed-form expression for the cdf of strength seems to be impossible (Le et al. 2011).

This paper presents two methods to calculate the strength cdf of general quasibrittle structures that fail at the macrocrack initiation from one RVE: (1) an approximate analytical formulation of the cdf of strength, which is developed on the basis of the Taylor series expansion of the grafted Weibull-Gaussian distribution; and (2) a general numerical scheme based on random placing of the RVEs, which resembles the Monte-Carlo type integration.

\section{Review of Weakest-Link Model and Its Nonlocal Extension}

This study focuses on the quasibrittle structures, which fail at the initiation of a macrocrack from one RVE. Statistically, the failure of this class of structures is equivalent to a chain of RVEs and follows the weakest-link model, in which each link corresponds to one RVE and has a statistically independent strength. Based on atomistic fracture mechanics and a statistical multiscale transition model, it has been shown that the strength distribution of one RVE $P_{1}(\sigma)$ can be modeled as a Gaussian distribution onto which a power-law tail (Weibull distribution) is grafted from the far-left end at a point of probability $P_{g r} \approx 10^{-3}-10^{-4}$ (Bažant and Pang 2007; Bažant et al. 2009; Le et al. 2011)

$$
\begin{gathered}
P_{1}(\sigma)=r_{f}\left(1-e^{-\left\langle\sigma / s_{1}\right\rangle^{m}}\right) \approx r_{f}\left\langle\sigma / s_{1}\right\rangle^{m}=P_{W}(\sigma) \quad\left(\sigma \leq \sigma_{g r}\right) \\
P_{1}(\sigma)=P_{g r}+\frac{r_{f}}{\delta_{G} \sqrt{2 \pi}} \int_{\sigma_{g r}}^{\sigma} e^{-\left(\sigma^{\prime}-\mu_{G}\right)^{2} / 2 \delta_{G}^{2}} d \sigma=P_{G}(\sigma) \\
\left(\sigma>\sigma_{g r}\right)
\end{gathered}
$$

where $\langle x\rangle=\max (x, 0) ; m=$ Weibull modulus; $s_{1}=s_{0} r_{f}^{1 / m} ; s_{0}=$ scale parameter of the Weibull tail; $\mu_{G}$ and $\delta_{G}=$ the mean and standard deviation, respectively, of the Gaussian core if considered extended to $-\infty ; r_{f}$ is a scaling parameter required to normalize the grafted cdf such that $P_{1}(\infty)=1 ; P_{g r}=$ grafting probability $=$ $r_{f}\left\{1-\exp \left[-\left(\sigma_{g r} / s_{1}\right)^{m}\right]\right\} ;$ and $\sigma_{g r}=$ grafting stress. Finally, continuity of the probability density function at the grafting point requires that $\left.\left(d P_{1} / d \sigma\right)\right|_{\sigma_{g r}^{+}}=\left.\left(d P_{1} / d \sigma\right)\right|_{\sigma_{g r}^{-}}$.

Based on the weakest-link model, the structure survives if and only if all the RVEs survive. Therefore, the strength distribution of the structure can be calculated by the following joint probability theorem:

$$
P_{f}\left(\sigma_{N}\right)=1-\prod_{i=1}^{N}\left\{1-P_{1}\left[\sigma_{N} s\left(\boldsymbol{x}_{i}\right)\right]\right\}
$$

or

$$
\ln \left[1-P_{f}\left(\sigma_{N}\right)\right]=\sum_{i=1}^{N} \ln \left\{1-P_{1}\left[\sigma_{N} s\left(\boldsymbol{x}_{i}\right)\right]\right\}
$$

where $N=$ number of RVEs in the structure $=V / V_{0} ; V$ and $V_{0}=$ the volumes of the entire structure and one RVE, respectively; $P_{f}=$ failure probability of structure $\left(1-P_{f}=\right.$ survival probability); and $\sigma_{N}=$ nominal strength of structure, which is a load parameter of the dimension of stress. In general, $\sigma_{N}=c_{n} P_{m} / b D$ or $c_{n} P_{m} / D^{2}$ for two-or three-dimensional scaling; $P_{m}=$ maximum load of the structure; $c_{n}=$ parameter chosen such that $\sigma_{N}$ represents the maximum principal stress in the structure; $b=$ structure thickness in the third dimension; $D=$ characteristic structure dimension or size; and $s\left(\boldsymbol{x}_{i}\right)=$ dimensionless stress field such that the actual stress $\sigma\left(\boldsymbol{x}_{i}\right)$ in the $i$-th RVE centered at coordinates $\boldsymbol{x}_{i}$ is equal to $\sigma_{N} s\left(\boldsymbol{x}_{i}\right) . \sigma\left(\boldsymbol{x}_{i}\right)$ may be interpreted as the maximum principal stress $\sigma_{I}$. 
More generally, stress $\sigma$ may be replaced by a suitable stress tensor function, $c(\boldsymbol{\sigma})$, capturing the triaxiality of failure criterion (Bažant and Planas 1998; Tsai and Wu 1971). This function can be simplified into the condition of independent survival of the RVE under each of the three principal stresses, $\sigma_{I}, \sigma_{I I}$, and $\sigma_{I I I}$ (Bažant and Xi 1991), provided that a crack normal to any principal stress can cause failure. In the following, the second and third principal stresses are assumed to be small compared with the first one, $\sigma\left(\boldsymbol{x}_{i}\right)=c\left[\boldsymbol{\sigma}\left(\boldsymbol{x}_{i}\right)\right] \approx \sigma_{I}\left(\boldsymbol{x}_{i}\right)$ :

$$
\begin{aligned}
\ln \left(1-P_{f}\right)= & \sum_{i=1}^{N} \ln \left(1-P_{1}\left\{c\left[\boldsymbol{\sigma}\left(\boldsymbol{x}_{i}\right)\right]\right\}\right) \\
& \approx \sum_{i=1}^{N} \sum_{j=I}^{I I I} \ln \left\{1-P_{1}\left[\sigma_{j}\left(\boldsymbol{x}_{i}\right)\right]\right\} \\
& \approx \sum_{i=1}^{N} \ln \left\{1-P_{1}\left[\sigma_{I}\left(\boldsymbol{x}_{i}\right)\right]\right\}
\end{aligned}
$$

It is clear that Eq. (4) requires the subdivision of a structure into a number of RVEs. In a general case, such a subdivision is subjective, typically nonunique, and often excessively refined. Therefore, it is preferable to replace the sum over a finite number of RVEs by an integral over the structure volume as follows:

$$
\ln \left(1-P_{f}\right)=\int_{V} \ln \left\{1-P_{1}\left[\sigma_{N} s(\boldsymbol{x})\right]\right\} \frac{d V(\boldsymbol{x})}{V_{0}}
$$

What governs the strength cdf of large-size structures is the farleft tail of the strength cdf of one RVE, i.e., $P_{1}(\sigma)=\left\langle\sigma / s_{0}\right\rangle^{m}$. Eq. (5) yields the following:

$$
P_{f}=1-\exp \left[-N_{e q}\left(\sigma_{N} / s_{0}\right)^{m}\right]
$$

and

$$
N_{e q}=\int_{V}\langle s(\boldsymbol{x})\rangle^{m} \frac{d V(\boldsymbol{x})}{V_{0}}
$$

By contrast, a closed-form expression of $P_{f}$ is not possible for small- and intermediate-size structures (i.e., Le et al. 2011). Therefore, one must still rely on the primary model, the weakest-link model [Eq. (5)].

For quasibrittle structures, the weakest-link model can further be generalized to include the nonlocality of the material due to its inhomogeneity (Bažant and Xi 1991; Bažant and Novák 2000). In such a generalization, the failure probability at a particular material point depends not only on the local stress but also on the stress in its neighborhood of a size approximately equal to the RVE size $l_{0}$. In deterministic calculations, the nonlocal concept is necessary for regularizing the boundary value problem with strainsoftening distributed damage and ensuring the convergence of fracture energy dissipation, which prevents spurious localization with failure at zero energy dissipation, and avoids spurious mesh sensitivity. In statistical calculations, nonlocal averaging is a convenient way to introduce a spatial correlation (Breysse and Fokwa 1992; Bažant and Novák 2000) and makes it possible to avoid direct use of the autocorrelation function.

The grafted Gauss-Weibull distribution of the RVE strength, derived from atomistic fracture mechanics and multiscale transition based on a hierarchy of series and parallel couplings, accounts for the statistical effect of material inhomogeneities (Bažant and Pang 2006, 2007; Bažant et al. 2009). However, standing alone, this distribution does not include the material characteristic length scale required for the finite weakest-link model. This characteristic length is introduced into the mathematical formulation [Eq. (4)] by associating the grafted distribution with the RVE size, and treating the structure as a system of discrete RVEs. In the continuum version with nonlocal averaging, the material characteristic length is imposed by making the effective size of the nonlocal averaging zone coincide with the RVE size. This effective size at the same time represents the autocorrelation length of the random strength field.

Following Bažant and Novák (2000), the nonlocal stress is calculated from the weighted average stress of the nonlocal zone as follows:

$$
\bar{\sigma}(\boldsymbol{x})=\int_{V} \alpha\left(\left\|\boldsymbol{x}-\boldsymbol{x}^{\prime}\right\|\right) \sigma\left(\boldsymbol{x}^{\prime}\right) d V\left(\boldsymbol{x}^{\prime}\right)
$$

where $\alpha=$ weighting function. There are various possible choices for an appropriate weighting function $\alpha$ (Bažant and Pijaudier-Cabot 1988; Bažant and Jirásek 2002), and the results do not depend too much on the choice (Bažant and Novák 2000). For the sake of simplicity, a stepwise constant weight function $\alpha$ is considered in the following:

$$
\propto\left(\boldsymbol{x}^{\prime}-\boldsymbol{x}\right)= \begin{cases}1 / V_{0} & \left(\left\|\boldsymbol{x}^{\prime}-\boldsymbol{x}\right\| \leq l_{0} / 2\right) \\ 0 & \left(\left\|\boldsymbol{x}^{\prime}-\boldsymbol{x}\right\|>l_{0} / 2\right)\end{cases}
$$

where $l_{0}=$ average size of RVE; and $V_{0}=\pi l_{0}^{2} / 4$ for twodimensional (2D) problems. Note that the nonlocal principal stresses should be calculated from the nonlocal stress tensor, which can be obtained by nonlocal averaging of each stress component (a direct averaging of the local principal stresses would not be correct because these stresses occur in different directions at every material point). Incorporating the nonlocal concept into the weakest-link model [Eq. (5)] yields the following:

$$
P_{f}=1-\exp \left(\int_{V} \ln \left\{1-P_{1}[\bar{\sigma}(\boldsymbol{x})]\right\} \frac{d V(\boldsymbol{x})}{V_{0}}\right)
$$

One important unsolved problem in the conventional nonlocal model is the ambiguity in the treatment of the weighting function when the nonlocal zone protrudes outside the structural boundary. Various approaches have been proposed, including these two:

1. Scale the part of weighting function that is inside the body so that the volume under the weighting function would revert to 1 (Bažant and Jirásek 2002); or

2. Use the original weighting function but add to it at some point a Dirac delta function of the same weight as the protruding part (Borino et al. 2003).

However, there is a lack of physical justifications for either approach.

In a recent conference article (Bažant et al. 2010), a nonlocal boundary layer model was proposed to overcome the ambiguity in adjusting the nonlocal weighting function when the averaging domain protrudes outside the boundary of the solid. In this model, the structure is divided into two parts: a boundary layer $V_{b}$ and an interior domain $V_{I}$. The boundary layer, which completely surrounds the interior domain, has a thickness equal to the RVE size $l_{0}$. In the interior domain, one can use the conventional nonlocal model to evaluate the stresses (Bažant and Novák 2000; Bažant and Jirásek 2002). Since the boundary layer thickness is equal to $l_{0}$, the nonlocal domain for the points in the interior domain will never protrude outside the structure boundary.

For the boundary layer, the continuum stress used to determine the failure probability $P_{f}$ is calculated from the deformation averaged over the thickness of the boundary layer $l_{0}$. Since, in most cases, the strain profile across the boundary layer is nearly linear, the averaged strain can approximately be taken as the continuum 

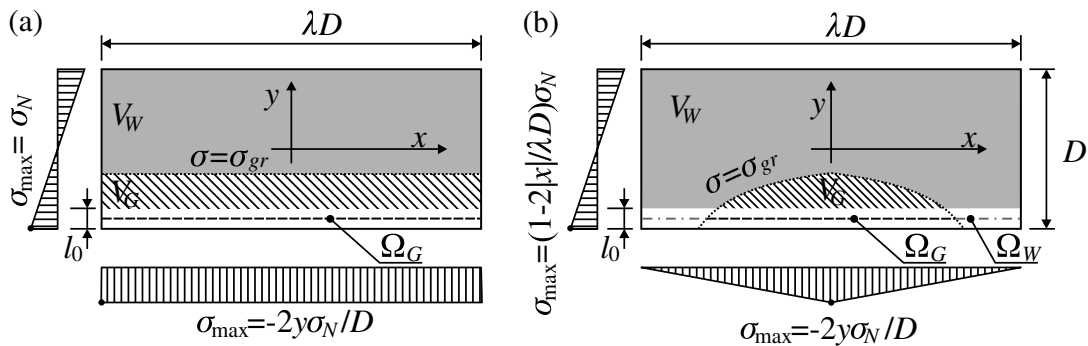

Fig. 1. Stress field in beam and its division into Weibullian and Gaussian regime: (a) pure bending; (b) three-point bending

strain at the middle surface $\Omega_{M}$ of the boundary layer. Therefore, the failure probability, as determined by the weakest-link model, Eq. (10), can be rewritten as

$$
\begin{aligned}
P_{f}=1-\exp ( & \int_{V_{I}} \ln \left\{1-P_{1}[\bar{\sigma}(\boldsymbol{x})]\right\} \frac{d V_{I}(\boldsymbol{x})}{V_{0}} \\
& \left.+l_{0} \int_{\Omega_{M}} \ln \left\{1-P_{1}[\sigma(\boldsymbol{x})]\right\} \frac{d \Omega_{M}(\boldsymbol{x})}{V_{0}}\right)
\end{aligned}
$$

Direct numerical integration of elastic stress field according to Eq. (11) will be referred to subsequently as the nonlocal boundary layer method.

Although the stress in the boundary layer is treated as local, it is actually a nonlocal stress since it corresponds to the average strain in the boundary layer, which is a feature encompassing spatial correlation. The difference from the nonlocal stress at the points of the interior domain is that it is defined only for boundary layer thickness as a whole and not for an arbitrary generic point across the thickness. Further note that the local treatment of the stress in the boundary layer has previously been advocated for the deterministic nonlocal models, to introduce diminishing nonlocality as the boundary is approached (Krayani et al. 2009). It has been shown that the proposed nonlocal boundary layer model agrees well with the original weakest-link model for structures with regular geometries, e.g., rectangular beams under pure bending (Bažant et al. 2010).

For very small structures for which the RVE size or the boundary layer exceeds about one-third of the cross-section dimension, the nonlocal concepts, including their present form with the boundary layer, lose physical meaning. In such cases, it is better to use the random discrete lattice-particle models. The geometrical randomness of discrete models is insufficient to simulate the size effect. To that end, one must include an autocorrelated random field of local strength, with the autocorrelation length equal to the RVE size (Carmeliet and de Borst 1995; Vořechovský and Sadílek 2008; Grassl and Bažant 2009).

For large-size structures, the boundary layer and the nonlocal zone have a negligible effect compared with the structure size effect. The nonlocal weakest-link model then converges to the local model [Eq. (6)].

\section{Approximate Closed-Form Expression for Strength Distribution}

In the present theory, the cdf of strength of one RVE is separated into two parts: a Weibull tail [Eq. (1a)], and a Gaussian core [Eq. (1b)]. Within the framework of nonlocal boundary layer model, one can likewise subdivide both the boundary layer $\Omega_{M}$ and the interior part $V_{I}$ into two parts:
1. The Weibullian region, where the principal stress is less than the grafting stress, causing the failure probability to be governed by the Weibull tail, Eq. (1a); and

2. The Gaussian region, where the principal stress is larger than the grafting stress, causing the failure probability to be governed by the Gaussian core, Eq. (1b). Fig. 1 shows such a division for beams under three-point bending and pure bending. Eq. (11) may then be rewritten as follows:

$$
\begin{aligned}
\ln \left(1-P_{f}\right)= & \underbrace{\int_{V_{W}} \ln \left\{1-P_{W}[\bar{\sigma}(\boldsymbol{x})]\right\} \frac{d V_{W}(\boldsymbol{x})}{V_{0}}}_{I_{V_{W}}} \\
& +\underbrace{\int_{V_{G}} \ln \left\{1-P_{G}[\bar{\sigma}(\boldsymbol{x})]\right\} \frac{d V_{G}(\boldsymbol{x})}{V_{0}}}_{I_{V_{G}}} \\
& +\underbrace{l_{0} \int_{\Omega_{W}} \ln \left\{1-P_{W}[\sigma(\boldsymbol{x})]\right\} \frac{d \Omega_{W}(\boldsymbol{x})}{V_{0}}}_{I_{\Omega_{W}}} \\
& +\underbrace{l_{0} \int_{\Omega_{G}} \ln \left\{1-P_{G}[\sigma(\boldsymbol{x})]\right\} \frac{d \Omega_{G}(\boldsymbol{x})}{V_{0}}}_{I_{\Omega_{G}}}
\end{aligned}
$$

where $V_{W}=$ Weibullian region of the interior part of structure = $\left\{\boldsymbol{x} \mid \boldsymbol{x} \in V_{I} \wedge \bar{\sigma}(\boldsymbol{x}) \leq \sigma_{g r}\right\} ; V_{G}=$ Gaussian region of the interior part of structure $=\left\{\boldsymbol{x} \mid \boldsymbol{x} \in V_{I} \wedge \bar{\sigma}(\boldsymbol{x})>\sigma_{g r}\right\} ; \Omega_{W}=$ Weibullian region of the boundary layer $=\left\{\boldsymbol{x} \mid \boldsymbol{x} \in \Omega_{M} \wedge \sigma(x) \leq \sigma_{g r}\right\}$; and $\Omega_{G}=$ Gaussian part of the boundary layer $=\left\{\boldsymbol{x} \mid \boldsymbol{x} \in \Omega_{M} \wedge \sigma(\boldsymbol{x})>\sigma_{g r}\right\}$. Clearly, one has $\quad V_{W} \cap V_{G}=\emptyset, \quad V_{W} \cup V_{G}=V_{I}, \quad \Omega_{W} \cap \Omega_{G}=\emptyset, \quad$ and $\quad \Omega_{W} \cup$ $\Omega_{G}=\Omega_{M}$.

The integrals for the Weibullian region can easily be computed by using the concept of $N_{e q}$ [Eq. (6)] as

$$
I_{V_{W}}=-\left(\frac{\sigma_{N}}{s_{0}}\right)^{m} N_{e q}^{V}
$$

where

$$
\begin{gathered}
N_{e q}^{V}=\int_{V_{W}}\langle\bar{s}(\boldsymbol{x})\rangle^{m} \frac{d V_{W}(\boldsymbol{x})}{V_{0}} \\
I_{\Omega_{W}}=-\left(\frac{\sigma_{N}}{s_{0}}\right)^{m} N_{e q}^{\Omega}
\end{gathered}
$$

where

$$
N_{e q}^{\Omega}=\int_{\Omega_{W}}\langle s(\boldsymbol{x})\rangle^{m} \frac{d \Omega_{W}(\boldsymbol{x}) l_{0}}{V_{0}}
$$


where $s(\boldsymbol{x})$ and $\bar{s}(\boldsymbol{x})=$ the dimensionless local and nonlocal stress fields for the Weibullian parts of the boundary layer and the interior part of structure, respectively. Note that the size of Weibullian regions $V_{W}$ and $\Omega_{W}$ explicitly depends on $\sigma_{N}$.

In contrast to integrals $I_{V_{W}}$ and $V_{\Omega_{W}}$ for the Weibullian region, closed-form expressions for integrals $I_{V_{G}}$ and $I_{\Omega_{G}}$ over the Gaussian region could not be obtained. The present method approximates the integrands of $I_{V_{G}}$ and $I_{\Omega_{G}}$ by means of the Taylor series expansion of the grafted Weibull-Gaussian distribution of the RVE strength.

The weakest-link model implies that the material elements with sufficiently small principal stress make negligible contributions to the failure of the entire structure. Thus, for the purpose of calculating the failure probability, one could neglect the part of structure that is subjected to relatively small stress. For example, consider the Weibull distribution with Weibull modulus $m=24$. If the failure probability of the element with principal stress $\sigma$ is denoted as $p$, then the failure probabilities of the elements with $0.8 \sigma, 0.6 \sigma$, and $0.4 \sigma$ are about $4.7 \times 10^{-3} p, 4.7 \times 10^{-6} p$, and $2.8 \times 10^{-10} p$, respectively, the last two being negligible.

Therefore, when evaluating integrals $I_{V_{G}}$ and $I_{\Omega_{G}}$, one can limit consideration to the elements in which the principal stress is within the range $\sigma \in\left[\mu \sigma_{N}, \sigma_{\max }\right]$, where $\sigma_{\max }=$ maximum principal stress used in the calculation and $\mu=\max \left(\sigma_{g r} / \sigma_{N}, 0.6 \sigma_{\max } / \sigma_{N}\right)$. For such a limited stress range, one can approximate the integrands of $I_{V_{G}}$ and $I_{\Omega_{G}}$ by the Taylor series expansion with respect to the maximum principal stress $\sigma_{\max }=t \sigma_{N}$.

The number of terms that must be retained in the Taylor series expansion depends on $\sigma_{N}, t, \mu$ and the parameters of the grafted Weibull-Gauss cdf. Using too many terms would defeat the goal of attaining a simple analytical solution. Thus, one can usually truncate the Taylor series expansion after its third derivative term. However, as will be shown subsequently, the truncation after the third derivative is insufficient for an accurate approximation of the function $\ln \left[1-P_{1}(\sigma)\right]$ for the range of $\sigma \in\left[\mu \sigma_{N}, t \sigma_{N}\right]$.

To further improve the approximation, one can consider a linear combination of the Taylor series expansions of $\ln \left[1-P_{1}(\sigma)\right]$ at $\sigma=\mu \sigma_{N}$ and $\sigma=t \sigma_{N}$ as follows:

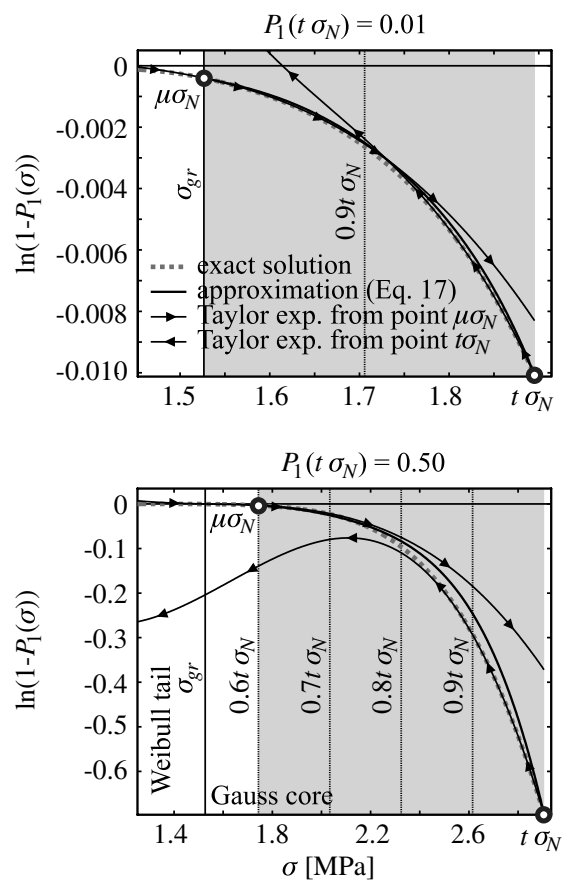

$$
\begin{aligned}
\ln \left[1-P_{1}(\sigma)\right]= & \omega(\sigma) \sum_{k=0}^{3} \frac{f^{(k)}\left(\mu \sigma_{N}\right)}{k !}\left(\sigma-\mu \sigma_{N}\right)^{k} \\
& +[1-\omega(\sigma)] \sum_{k=0}^{3} \frac{f^{(k)}\left(t \sigma_{N}\right)}{k !}\left(\sigma-t \sigma_{N}\right)^{k}
\end{aligned}
$$

where $f^{(k)}(\sigma)=d^{k} \ln \left[1-P_{G}(\sigma)\right] / d \sigma^{k}$; and the detailed expressions for $f^{(k)}(\sigma)$ are given in the appendix. To make the approximated function match the asymptotic properties of function $\ln \left[1-P_{1}(\sigma)\right]$ at $\sigma=\mu \sigma_{N}$ and $\sigma=t \sigma_{N}$, it is clear that the function $\omega(\sigma)$ must decay from 1 at $\sigma=\mu \sigma_{N}$ to 0 at $\sigma=t \sigma_{N}$. The present method introduces the following quadratic function:

$$
\omega(\sigma)=1-\left[\frac{\sigma-\mu \sigma_{N}}{t \sigma_{N}-\mu \sigma_{N}}\right]^{2}
$$

The performance of this approximation is evaluated for different values of $t \sigma_{N}$ corresponding to the failure probabilities $P_{1}\left(t \sigma_{N}\right)=0.01,0.10,0.50$, and 0.99 . Fig. 2 presents the comparison between the exact solution of $\ln \left[1-P_{1}(\sigma)\right]$, the three-term Taylor series expansion of $\ln \left[1-P_{1}(\sigma)\right]$ at $\sigma=t \sigma_{N}$, the three-term Taylor series expansion of $\ln \left[1-P_{1}(\sigma)\right]$ at $\sigma=\mu \sigma_{N}$, and the present approximation [Eqs. (17) and (18)] for the stress range $\sigma \in\left[\mu \sigma_{N}, t \sigma_{N}\right]$. It can be seen that the three-term Taylor series expansion at either $\sigma=t \sigma_{N}$ or $\mu \sigma_{N}$ is unable to provide a close approximation [Eqs. (17) and (18)]. In contrast, the proposed approximation function agrees well with the exact value of $\ln \left[1-P_{1}(\sigma)\right]$. Consequently, integrals $I_{V_{G}}$ and $I_{\Omega_{G}}$ can be rewritten as

$$
\begin{aligned}
I_{V_{G}}(t, \mu)= & \sum_{k=0}^{3} \frac{\sigma_{N}^{k}}{k !} f^{(k)}\left(\mu \sigma_{N}\right) \Delta_{V_{G}, 1}(k, t, \mu) \\
& +\sum_{k=0}^{3} \frac{\sigma_{N}^{k}}{k !} f^{(k)}\left(t \sigma_{N}\right) \Delta_{V_{G}, 2}(k, t, \mu)
\end{aligned}
$$

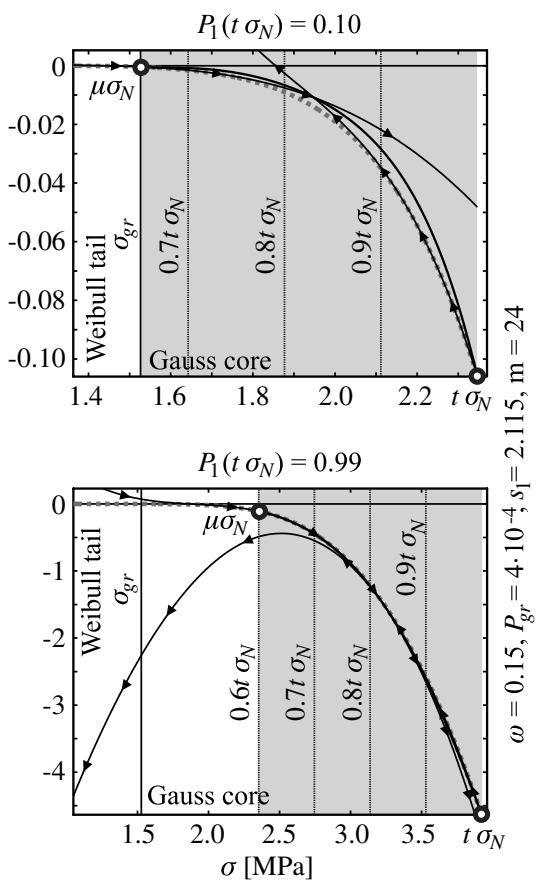

Fig. 2. Approximation of $\ln \left[1-P_{1}(\sigma)\right]$ by Taylor series expansion 


$$
\begin{aligned}
I_{\Omega_{G}}(t, \mu)= & \sum_{k=0}^{3} \frac{\sigma_{N}^{k}}{k !} f^{(k)}\left(\mu \sigma_{N}\right) \Delta_{\Omega_{G}, 1}(k, t, \mu) \\
& +\sum_{k=0}^{3} \frac{\sigma_{N}^{k}}{k !} f^{(k)}\left(t \sigma_{N}\right) \Delta_{\Omega_{G}, 2}(k, t, \mu)
\end{aligned}
$$

where:

$$
\Delta_{V_{G}, 1}(k, t, \mu)=\frac{1}{V_{0}} \int_{V_{G}(t, \mu)}\left\{1-\left[\frac{\bar{s}(\boldsymbol{x})-\mu}{t-\mu}\right]^{2}\right\}[\bar{s}(\boldsymbol{x})-\mu]^{k} d V_{G}
$$

$$
\begin{aligned}
& \Delta_{V_{G}, 2}(k, t, \mu)=\frac{1}{V_{0}(t-\mu)^{2}} \int_{V_{G}(t, \mu)}[\bar{s}(\boldsymbol{x})-\mu]^{2}[\bar{s}(\boldsymbol{x})-t]^{k} d V_{G} \\
& \Delta_{\Omega_{G}, 1}(k, t, \mu)=\frac{l_{0}}{V_{0}} \int_{\Omega_{G}(t, \mu)}\left\{1-\left[\frac{s(\boldsymbol{x})-\mu}{t-\mu}\right]^{2}\right\}[s(\boldsymbol{x})-\mu]^{k} d \Omega_{G} \\
& \Delta_{\Omega_{G}, 2}(k, t, \mu)=\frac{l_{0}}{V_{0}(t-\mu)^{2}} \int_{\Omega_{G}(t, \mu)}[s(\boldsymbol{x})-\mu]^{2}[s(\boldsymbol{x})-t]^{k} d \Omega_{G}
\end{aligned}
$$

For structures with some simple stress fields, such as the linear and bilinear stress profiles, $\Delta_{V_{G, i}}(k, t, \mu)$ and $\Delta_{\Omega_{G, i}}(k, t, \mu)$ $(i=1,2)$ can be integrated analytically. This is likely possible for many structures, for which the stress field near the point with the largest maximum principal stress can be approximated by a linear or bilinear function.

\section{Computation of Strength Distribution by Pseudorandom Placing of RVEs}

For complicated structures, the stress field in the region near the maximum principal stress point could be highly nonlinear. In such a case, the use of the aforementioned Taylor series expansion method would not lead to a closed-form expression. One must then resort to some numerical method to calculate the cdf of structural strength. So, besides the use of Eq. (11), the present study proposes a general numerical scheme based on a pseudorandom placing of the RVEs, referred to as the direct RVE placing method. This method directly corresponds to the original weakest-link model [Eq. (3)].

There are many possible ways of placing the RVEs in the structure, and each of them would yield a different strength cdf. Therefore, for statistically isotropic materials, it is preferable to make repeated pseudorandom choices of the RVE locations, and then average the results of all the realizations. The main advantage of the pseudorandom placing of the RVEs is that it eliminates the directional bias, which inevitably arises with a regular placing. For small-size structures where the RVE size is comparable to the structure size, one would need to generate many realizations of the random placing of RVEs. For large-size structures where the RVE size is negligible compared with the structure size, the RVE converges to a point in the structure and the random placing of RVEs would not have a significant effect on the resulting cdf of strength. Therefore, fewer realizations are needed for large-size structures.

The present method employs the centroidal Voronoi tessellation to generate a set of random locations of $N$ points, where $N=V / V_{0}$. Each of these points represents the center of one RVE. This tessellation produces a set of points corresponding to the centroids of the Voronoi cells, which satisfy two essential requirements:

1. The distance between any two adjacent points is approximately equal to the RVE size $l_{0}$; and

2. The minimum distance of the point from the structure boundary is close to $l_{0} / 2$ (Fig. 3 ).

Once the centers of the assumed RVEs are fixed, the average stress for each RVE can be obtained based on the stress field calculated by the standard finite element method. The RVE center $\rho$ generally does not coincide with the integration point of the finite elements, $\boldsymbol{x}$. So the nonlocal stress for each RVE is calculated by averaging the stresses at the integration points that are enclosed within the RVE (see Fig. 4), i.e.,

$$
\bar{\sigma}\left(\boldsymbol{\rho}_{i}\right)=\frac{\sum_{j=1}^{N} \sigma\left(\boldsymbol{x}_{j}\right) \alpha\left(\boldsymbol{x}_{j}-\boldsymbol{\rho}_{i}\right) \kappa_{j}}{\sum_{j=1}^{N} \alpha\left(\boldsymbol{x}_{j}-\boldsymbol{\rho}_{i}\right) \kappa_{j}}
$$

where $\alpha\left(\boldsymbol{x}_{j}-\boldsymbol{\rho}_{i}\right)=$ weighting function; and $\kappa_{j}=$ the volume associated with integration point $\boldsymbol{x}_{j}$. To compute the average stress in one RVE, consider only the integration points that lie within that RVE. Therefore, the function $\alpha$ must have a cut-off that excludes all the integration points outside the RVE, i.e., $\alpha=0$, if $\left\|\boldsymbol{x}_{j}-\boldsymbol{\rho}_{i}\right\|>l_{0} / 2$. For the sake of simplicity, a piecewise constant function [Eq. (9)] can be used. By combining the weakest-link model with the centroidal Voronoi tessellation, the cdf of structural strength can be calculated as follows:

$$
\ln \left(1-P_{f}\right)=\sum_{i=1}^{N} \ln \left\{1-P_{1}\left[\bar{\sigma}\left(\rho_{i}\right)\right]\right\}
$$

Note that this approach closely resembles the Monte-Carlo integration of Eq. (5) with a fixed number $N$ of integration points (e.g., Dimov 2008), although the present method of placing the integration points is more refined.

Centroidal Voronoi Tesselation

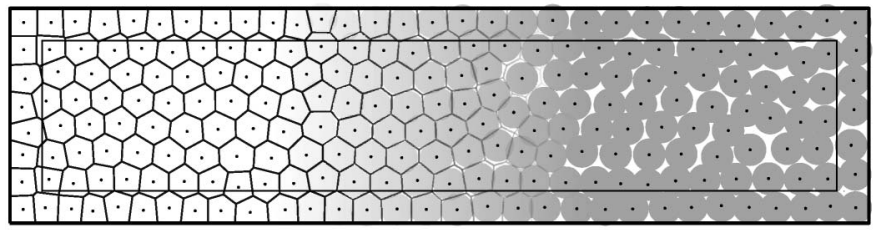

pseudo-randomly placed RVEs

Fig. 3. Example of centroidal Voronoi tessellation (left) and obtained imaginary locations of RVEs (right)

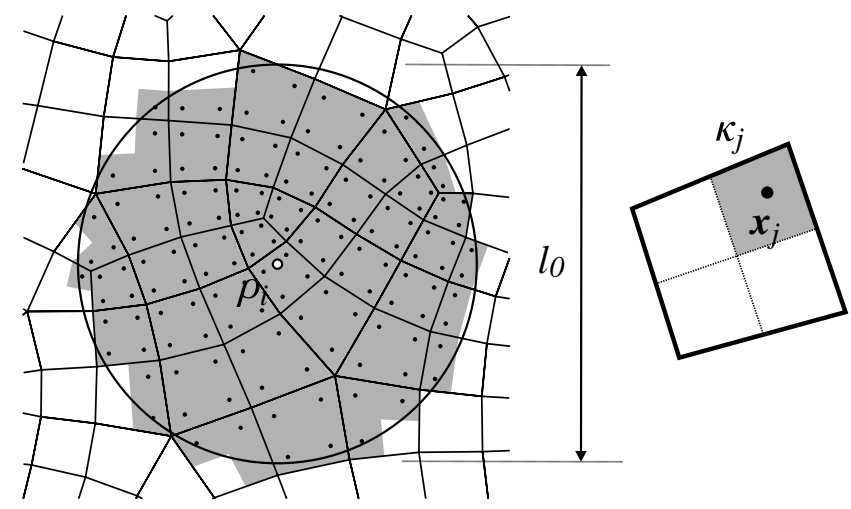

Fig. 4. Averaging of stress obtained by FEM in imaginary $i$-th RVE volume 
In the centroidal Voronoi tessellation, there is always a layer of RVEs placed along the structure boundary (Fig. 3), which agrees with the concept of the nonlocal boundary layer model. One difference, though, is that the nonlocal boundary layer model uses the stress at the middle surface of the layer while the current approach uses the average stress across the RVE. Therefore, in the nonlocal boundary layer model, switching between the cubical RVE shape, $V_{0}=l_{0}^{3}$ (squares $V_{0}=l_{0}^{2}$ ) and the spherical one, $V_{0}=\pi l_{0}^{3} / 6$ (circles $V_{0}=\pi l_{0}^{2} / 4$ ), has no effect, while in the direct RVE placing method, the RVE shape affects the number $N$ of the RVEs and consequently their mutual distances as well as the minimal distance to the boundary.

The present study uses spherical RVEs. Since an assembly of non-overlapping spheres would occupy a volume larger than the structure volume $V$, the spheres must overlap each other to fit this assembly into the structure volume $V$. Thus the mutual average distance between the RVE centers must be slightly less than $l_{0}$. It also affects the distance between the centers of circumferential RVEs and the structure boundary, which would again be slightly less than $l_{0} / 2$. Therefore, in general, the present method predicts higher failure probabilities than the nonlocal boundary layer method does.

\section{Analysis of Failure Statistics of Quasibrittle Structures}

\section{Beams under Three-Point Bending and Pure Bending}

Three-point and four-point bending tests are commonly adopted to measure the strength histograms of quasibrittle structures (Weibull 1939; Munz and Fett 1999; Tinschert et al. 2000; Lohbauer et al. 2002; dos Santos et al. 2003). For beams with a large span-to-depth ratio, the engineering beam theory may be used to calculate the maximum principal stress field. Accordingly, for three-point bending tests, the stress field can be represented by a two-way linear stress gradient along both the beam depth and span.
In four-point bending tests in which the two loading points are far apart, the failure is governed by the middle portion between the loading points, which is essentially experiencing a constant bending moment, i.e., pure bending. The stress field of the middle portion of the beam can be represented by a one-way stress gradient along the beam depth. Nevertheless, if the two loading points are not far apart, the contribution of the end portion of beam between the loading point and the support, which has a two-way linear stress distribution, can simply be added.

For the case of pure bending, the stress varies linearly in the $y$-direction (normal to the beam axis). Consider a prismatic beam of depth $D$ (y-direction) and length $\lambda D$ (x-direction). 2D analysis presumes that the failure occurs simultaneously along the whole prism thickness (in the $z$-direction). The elastic stress in the body can be described as

$$
\sigma(\boldsymbol{x})=\sigma_{x}(x, y)=-\frac{2 y}{D} \sigma_{N}
$$

Since the stress varies linearly, the nonlocal principal stress field coincides with the local principal stress field. The maximum stress in the boundary layer is $\sigma_{\max }^{\Omega}=\left(1-l_{0} / D\right) \sigma_{N}$ and the maximum stress in the interior part is $\sigma_{\max }^{V}=\left(1-2 l_{0} / D\right) \sigma_{N}$. Within the framework of aforementioned Taylor series expansion method, the boundary between the Weibull and Gaussian regions is given by the straight line $y=-\sigma_{g r} D /\left(2 \sigma_{N}\right)$ [Fig. 1(a)]. For the Taylor series expansion method, the following quantities are needed:

$$
\begin{gathered}
N_{e q}^{V}=\frac{\lambda D^{2}}{2(m+1) V_{0}} \min \left\{\frac{\sigma_{g r}}{\sigma_{N}}, 1-\frac{2 l_{0}}{D}\right\}^{m+1} \\
N_{e q}^{\Omega}=\left\{\begin{array}{l}
\frac{\lambda D l_{0}}{V_{0}}\left(1-\frac{l_{0}}{D}\right)^{m} \\
0 \text { otherwise }
\end{array} \quad\left(1-\frac{l_{0}}{D} \leq \frac{\sigma_{g r}}{\sigma_{N}}\right)\right.
\end{gathered}
$$


where $\alpha_{i}=$ coefficients of binomial expansion of $(x-\mu)^{2}(x-t)^{k}$. Substituting Eqs. (28)-(33) into Eqs. (12), (19), and (20), one can calculate the corresponding strength cdf analytically.

For beams under three-point bending, the stress field $\sigma(x, y)$ can be assumed to be linear in two directions (Fig. 1(b)). The stress field can be written as

$$
\sigma=\frac{2 y}{D}\left(\frac{2|x|}{D \lambda}-1\right) \sigma_{N}
$$

Similar to the case of pure bending, the quantities that are required for the Taylor series expansion method can be obtained as follows:

$$
\begin{aligned}
& N_{e q}^{V}=\frac{\lambda D^{2} \min \left\{\frac{\sigma_{g r}}{\sigma_{N}}, 1-\frac{2 l_{0}}{D}\right\}^{m+1}}{2(m+1)^{2} V_{0}}\left[1-(m+1) \ln \left(\frac{D \min \left\{\frac{\sigma_{g r}}{\sigma_{N}}, 1-\frac{2 l_{0}}{D}\right\}}{D-2 l_{0}}\right)\right] \\
& N_{e q}^{\Omega}=\frac{\lambda D^{2} \min \left\{\frac{\sigma_{g r}}{\sigma_{N}}, 1-\frac{l_{0}}{D}\right\}^{m+1} l_{0}}{\left(D-l_{0}\right)(m+1) V_{0}} \\
& \Delta_{V_{G}, 1}(k, t, \mu)=\left\{\begin{array}{l}
\frac{D^{2} \lambda}{2 V_{0}}\left[f(k)-\frac{1}{(t-\mu)^{2}} f(k+2)\right] \quad\left(1-\frac{2 l_{0}}{D}>\mu\right) \\
0 \quad \text { otherwise }
\end{array}\right. \\
& \Delta_{V_{G}, 2}(k, t, \mu)=\left\{\begin{array}{l}
\frac{1}{V_{0}(t-\mu)^{2}} \sum_{i=0}^{k+2} \alpha_{i} g(i) \quad\left(1-\frac{2 l_{0}}{D}>\mu\right) \\
0 \quad \text { otherwise }
\end{array}\right. \\
& \Delta_{\Omega_{G}, 1}(k, t, \tau)=\left\{\begin{array}{l}
\frac{\lambda D^{2} l_{0}}{\left(D-l_{0}\right) V_{0}}\left[\frac{1}{k+1}\left(1-\frac{l_{0}}{D}-\mu\right)^{k+1}-\frac{1}{(k+3)(t-\mu)^{2}}\left(1-\frac{l_{0}}{D}-\mu\right)^{k+3}\right]\left(1-\frac{l_{0}}{D}>\frac{\sigma_{g r}}{\sigma_{N}}\right) \\
0 \quad \text { otherwise }
\end{array}\right. \\
& \Delta_{\Omega_{G}, 2}(k, t, \mu)=\left\{\begin{array}{l}
\frac{(-1)^{k+2} \lambda D^{2} l_{0}}{2(t-\mu)^{2}\left(D-l_{0}\right) V_{0}} \sum_{i=0}^{k+2} \frac{\beta_{i}}{i+1}\left(\frac{(1-\mu) D-l_{0}}{2 D}\right)^{i+1} \quad\left(1-\frac{l_{0}}{D}>\frac{\sigma_{g r}}{\sigma_{N}}\right) \\
0 \quad \text { otherwise }
\end{array}\right.
\end{aligned}
$$

where $\beta_{i}=$ coefficients of the binomial expansion of $\left[x+\mu+\left(l_{0}-D\right) / D^{2} \lambda\right]^{2}\left[x+t+\left(l_{0}-D\right) / D^{2} \lambda\right]^{k}$, and

$$
f(k)=\frac{(-1)^{k}}{k+1}\left\{\mu^{k+1} \ln \left(\frac{D \mu}{D-2 l_{0}}\right)+\sum_{i=0}^{k} t^{i} \frac{-\left(2 l_{0} / D-1+\mu\right)^{k-i+1}}{k-i+1}\right\}
$$

$$
\begin{aligned}
g(i)= & \frac{\lambda \mu^{i+1} D^{2}}{4(i+1)} \ln \left[1-2 D(1-\mu)+4 l_{0}\right] \\
& -\frac{(-1)^{i+1}}{(i+1)^{2}}\left(\frac{2}{D}\right)^{k-1}\left(l_{0}-D / 2\right)^{i+1} \\
& \times\left\{\left[4 l_{0}+1-2 D(1-\mu)\right]^{i+1}-1\right\}
\end{aligned}
$$

For the interior region, the present method uses the local stress to approximate the nonlocal stress, which is exact for the most part of the structure except for the midspan. Such an approximation will not cause any error in the calculation of the cdf of both the smallsize and large-size beams because: (1) for small-size beams, the failure probability is entirely governed by the boundary layer; and (2) for large-size beams, the nonlocal stress field converges to the local stress field. For intermediate-size beams, which lie between these two extreme cases, the error in calculating the cdf of strength is expected to be insignificant.

Figs. 5 and 6 present the comparison of the calculated strength cdfs of beams under three-point and four-point bending by using the Taylor series expansion method, the direct RVE placing method, and the nonlocal boundary layer method. For the fourpoint bend beam, the two loading points are placed at a quarter length from the supports. Consider a linear one-way stress distribution for the middle portion of the beam, and a linear two-way stress distribution for the two end portions of the beam. In the calculation, fix the beam dimension, $D=0.1 \mathrm{~m}$ and $\lambda=4$, and consider four different RVE sizes $l_{0}=3.75,7.5,15$, and 


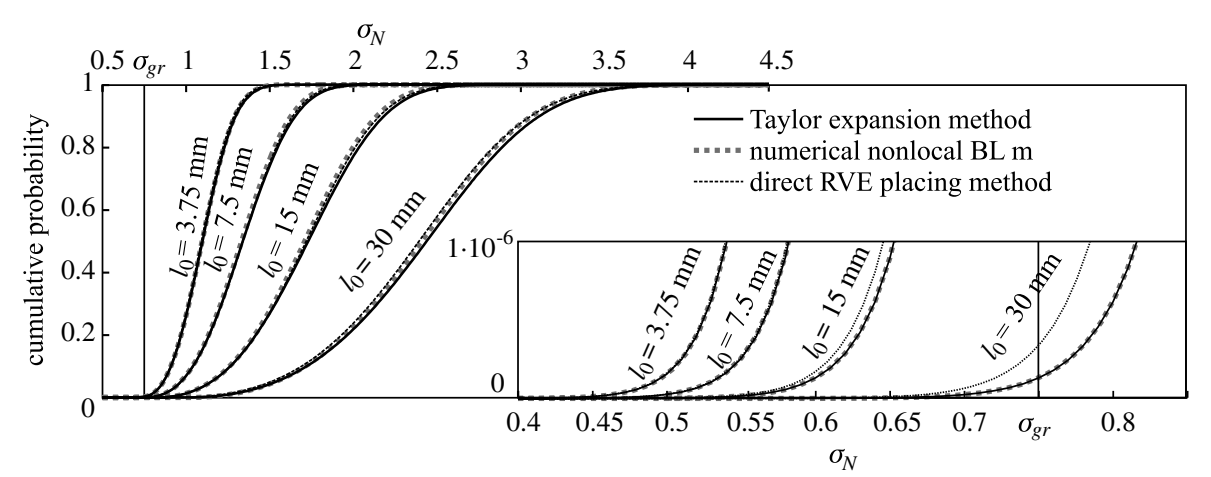

Fig. 5. Comparison of cdfs of four geometrically similar beams under three-point bending

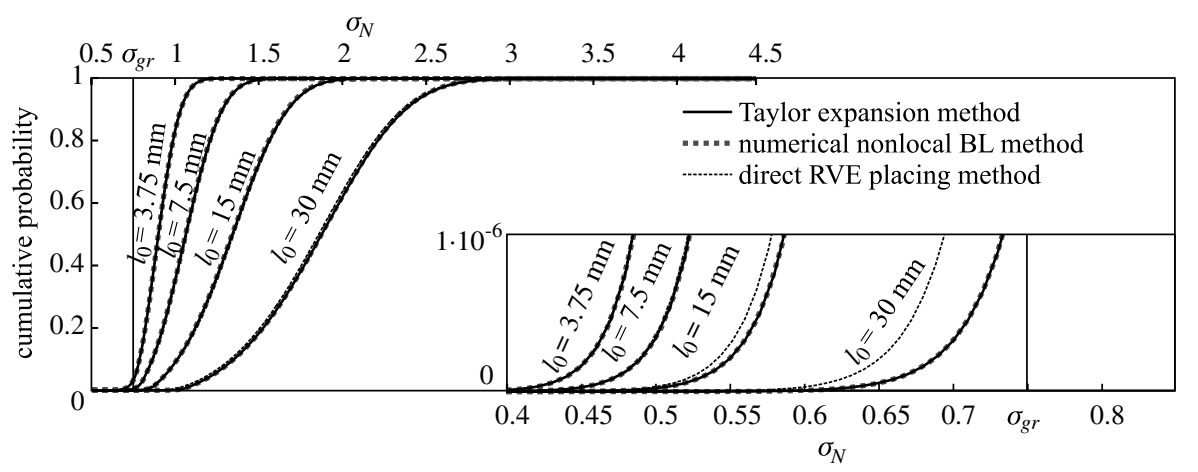

Fig. 6. Comparison of cdfs of four geometrically similar beams under four-point bending

(a)

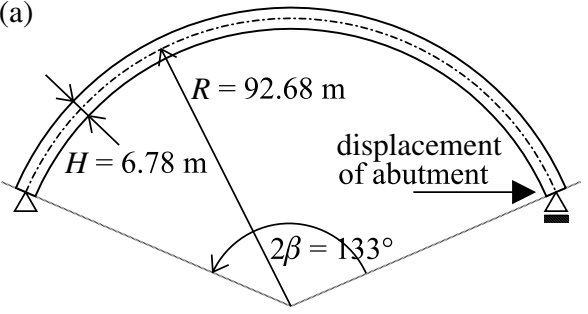

(b)

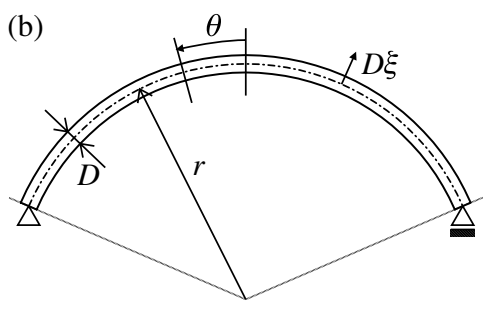

Fig. 7. Simplified 2D model of Malpasset dam

$30 \mathrm{~mm}$. The statistical parameters used in the calculations are as follows: $s_{1}=1 \mathrm{MPa}, m=24, \mu_{G}=2.0956 \mathrm{MPa}$, and $\delta_{G}=$ $0.5361 \mathrm{MPa}$. For the direct RVE placing method, 10, 50, 100, and 100 simulations are performed for beams with RVE size $l_{0}=3.75,7.5,15$, and $30 \mathrm{~mm}$, respectively, and the calculated cdfs are averaged for each size.

As seen from Figs. 5 and 6, the strength cdfs calculated by the Taylor series expansion method and the nonlocal boundary layer method agree with each other for all the sizes. The direct RVE placing method predicts a higher failure probability because the average distance to the boundary is smaller than $l_{0} / 2$, which implies that the stresses in the boundary RVEs are higher than the stresses in the center of the boundary layer.

The aforementioned calculations demonstrate that the Taylor series expansion method can accurately calculate the failure probability. Note that the present formulation is generally applicable to the strength cdf of structures whose critical stress region, i.e., $\sigma \in\left[\mu \sigma_{\max }, t \sigma_{\max }\right]$, has approximately a linear one-way or two-way stress profile.

\section{Example: Analysis of Failure Statistics of Malpasset Dam}

The Malpasset dam in the French Maritime Alps was built in 1954 and failed at its first complete filling in 1959 (Bartle 1985; Levy and Salvadori 1992; Pattison 1998). It is believed that the failure was caused by vertical flexural cracks engendered by lateral displacement of abutment because of a slip of thin clay-filled seam in schist. Following the previous study (Bažant et al. 2007), one could model the midheight cross-section of dam as a horizontal circular two-hinge arch of constant depth $H=6.78 \mathrm{~m}$. The radius and central angle of the arch are $R=92.68 \mathrm{~m}$ and $2 \beta=133^{\circ}$. The dam is loaded by an enforced displacement caused by slip of its right support (Fig. 7). 


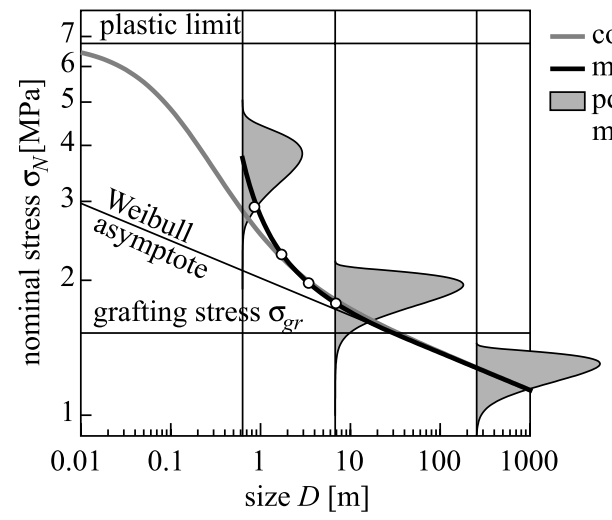

Fig. 8. Comparison of mean size effect curves including probability density functions of three different dam sizes

Stochastic finite element simulation in the previous study (Bažant et al. 2007) demonstrated a strong energetic-statistical size effect on the strength of the dam. The present study uses the numerical nonlocal boundary layer method, the Taylor series expansion method, and the direct RVE placing method to investigate the failure statistics of the dam and the corresponding size effect on its mean strength.

To examine the size effect on the failure statistics, consider geometrically similar arches where $D=$ depth of the arch, which is considered as the characteristic size to be scaled, and $r=$ radius of arch $=13.67 D$. For such a $2 \mathrm{D}$ arch model, it is convenient to express the uniaxial stress in the polar coordinates $\xi, \theta$. Consider that the right support of the arch is subjected to a horizontal slip $u$. From Castigliano's theorem and the classical theory of bending, the bending stress in the arch can be calculated as

$$
\sigma=\sigma_{N} \frac{2(\cos \beta-\cos \theta) \xi}{1-\cos \beta}
$$

where

$$
\sigma_{N}=\frac{6 u(1-\cos \beta)}{C b D} \frac{r}{D}
$$

Here $C=$ compliance $=\left[\int_{-\beta}^{\beta}(\cos \theta-\cos \beta)^{2} d \theta\right] r^{3} / E ; E=$ elastic modulus; $\xi=y / D$; and $y=$ distance from the neutral axis; and $\sigma_{N}$ is the maximal tensile stress at the boundary $(\xi=-1 / 2)$ of the central cross-section $(\theta=0)$.

The nonlocal boundary layer method is first used to calculate the strength cdf of the arch structure, where the elastic stress field is taken from an elastic finite element analysis. The RVE size $l_{0}$ is taken to be $0.28 \mathrm{~m}$ (Bažant et al. 2007). Considering different arch sizes, one can obtain the size effect curve of the mean strength. The present study uses the following statistical parameters for the cdf of RVE strength: $s_{1}=2.115 \mathrm{MPa}, m=24, \mu_{G}=2.9053 \mathrm{MPa}$, and $\delta_{G}=0.4369 \mathrm{MPa}$.

The mean size effect curve calculated by the nonlocal boundary layer method matches well the mean size effect calculated by using the microplane model (Bažant et al. 2007) for intermediate- and large-size arches (Fig. 8). These two models begin to deviate when there are only 3-4 RVEs across the arch thickness. This is because, for small-size structures, the boundary layer occupies too large a portion of the thickness. The averaging of elastic strain within the boundary layer cannot realistically represent the actual stress and strain redistribution in deterministic calculation.

The cdf of the arch strength can also be calculated by the Taylor series expansion method. Based on the analytical stress field [Eq. (43)] with the small-angle approximation $\cos \theta=1+\theta^{2} / 2$,

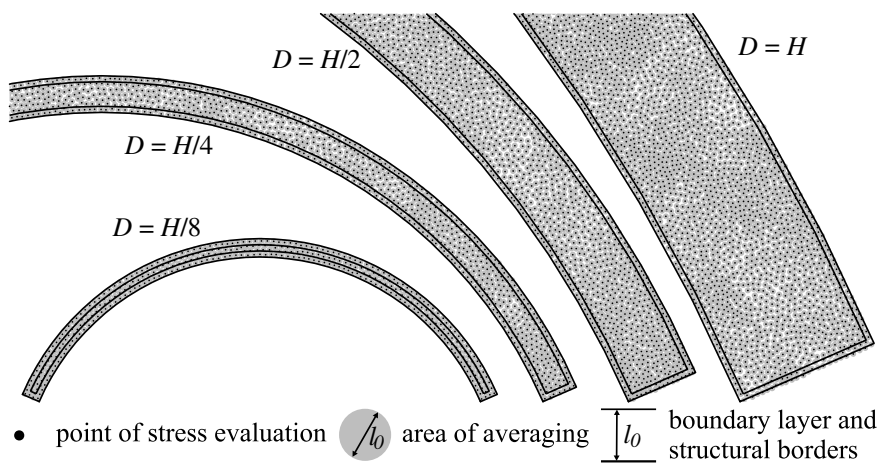

Fig. 9. Example of pseudorandom location of RVEs in dam obtained by centroidal Voronoi tessellation for four sizes $D$

one can obtain closed-form expressions for $N_{e q}^{V}, N_{e q}^{\Omega}, \Delta_{V_{G}}$ and $\Delta_{\Omega_{G}}$. The detailed expressions (which are omitted here) yield an analytical equation for the cdf of the arch strength.

Finally, the direct RVE placing method is used to calculate the cdfs of strength of geometrically similar arches for four different sizes: $D=H, H / 2, H / 4$, and $H / 8$. The arch volume is filled by the RVEs based on the centroidal Voronoi tessellation. For arches with $D=H, H / 2, H / 4$, and $H / 8,3,30,50$, and 50 realizations are performed, respectively, and the average cdf of nominal strength is calculated. Fig. 9 presents typical RVE placings for arches of each size. The stress field from an elastic finite element analysis is used to compute the average stress for each RVE [Eq. (25)]. The weakest-link model then yields the cdf of structure strength.

Fig. 10 presents the cdfs of structural strength calculated by the nonlocal boundary layer model, by the Taylor series expansion method, and by the direct RVE placing method. Here, the nonlocal boundary layer model agrees well with the Taylor series expansion method because the stress obtained by elastic finite element analysis is very close to the engineering stress field from Eq. (43). Again, the direct RVE placing method predicts a higher failure probability because of the fact that the distance of the circumferential RVEs from the boundary is smaller than $l_{0} / 2$. For the scaled large-size arches, for which the RVE size becomes negligible compared with the arch size, all the three methods converge to essentially the same cdf. 


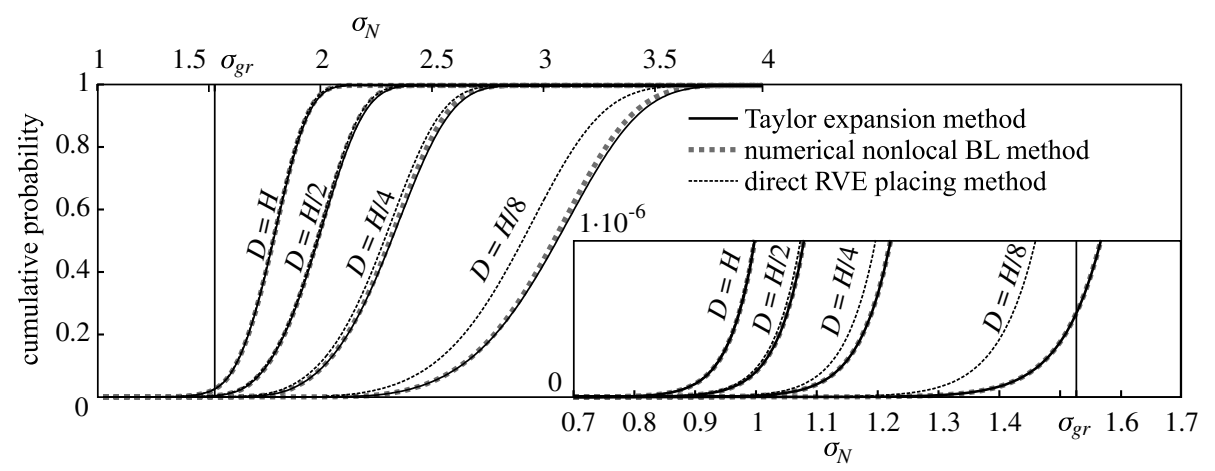

Fig. 10. Comparison of cdfs of four geometrically similar dams (arches)

\section{Summary and Conclusions}

This study proposes an approximate analytical formulation for the strength distribution of quasibrittle structures failing at the initiation of a macrocrack from one RVE. This formulation yields a closedform expression of strength distribution for structures in which the stress distribution in the high stress region can be approximated by some simple functions. The formulation agrees well with two numerical approaches, namely, the random RVE placing method and the nonlocal boundary layer method. The closed-form expression for the strength distribution provides an efficient way to calibrate the statistical parameters by optimum fitting of the observed strength histograms, and it also creates a basis for the analytical formulation of size- and geometry-dependent safety factors.

\section{Appendix. Derivatives of $P_{1}(\sigma)$ and $\ln \left[1-P_{1}(\sigma)\right]$}

Derivatives of the grafted Weibull-Gaussian distribution $P_{1}(\sigma)$ are as follows:

$$
\begin{gathered}
p_{1}(\sigma)=\frac{\partial P_{1}(\sigma)}{\partial \sigma}= \begin{cases}p_{W}=r_{f} \frac{m}{s}\left\langle\sigma / s_{1}\right\rangle^{m-1} e^{-\left\langle\sigma / s_{1}\right\rangle^{m}}, & \sigma \leq \sigma_{g r} \\
p_{G}=r_{f} \frac{1}{\sqrt{2 \pi \delta_{G}}} e^{-\frac{\left(x-\mu_{G}\right)^{2}}{2 \delta_{G}^{2}},} & \sigma>\sigma_{g r}\end{cases} \\
p_{1}^{\prime}(\sigma)=\frac{\partial p_{1}(\sigma)}{\partial \sigma}= \begin{cases}r_{f}\left(\frac{m}{s_{1}}\right)^{2}\left\langle\frac{\sigma}{s_{1}}\right\rangle^{m-2} e^{\left\langle-\frac{\sigma}{\left.s_{1}\right)^{m}}\right.}\left[1-\frac{1}{m}-\left\langle\sigma / s_{1}\right\rangle^{m}\right], & \sigma \leq \sigma_{g r} \\
-r_{f} \frac{x-\mu_{G}}{\delta_{G}^{3} \sqrt{2 \pi}} e^{-\frac{\left(x-\mu_{G}\right)^{2}}{2 \delta_{G}^{2}}}, & \sigma>\sigma_{g r}\end{cases} \\
p_{1}^{\prime \prime}(\sigma)=\frac{\partial^{2} p_{1}(\sigma)}{\partial \sigma^{2}}= \begin{cases}r_{f}\left(\frac{m}{s_{1}}\right)^{3}\left\langle\frac{\sigma}{s_{1}}\right\rangle^{m-3} e^{\left.-\frac{\langle\sigma}{s_{1}}\right\rangle^{m}}\left[1-\frac{3}{m}+\frac{2}{m^{2}}+\left(\frac{3}{m}-3\right)\left\langle\frac{\sigma}{s_{1}}\right\rangle^{m}+\left\langle\sigma / s_{1}\right\rangle^{2 m}\right], & \sigma \leq \sigma_{g r} \\
-r_{f} \frac{1}{\delta_{G}^{3} \sqrt{2 \pi}} e^{-\frac{\left(x-\mu_{G}\right)^{2}}{2 \delta_{G}^{2}}}\left[1-\frac{2\left(x-\mu_{G}\right)^{2}}{2 \delta_{G}^{2}}\right], & \sigma>\sigma_{g r}\end{cases}
\end{gathered}
$$

Derivatives of $\ln \left[1-P_{1}(\sigma)\right]$ are as follows:

$$
\begin{gathered}
\frac{\partial \ln \left[1-P_{1}(\sigma)\right]}{\partial \sigma}=-\frac{p_{1}(\sigma)}{1-P_{1}(\sigma)} \\
\frac{\partial^{2} \ln \left[1-P_{1}(\sigma)\right]}{\partial \sigma^{2}}=-\left[\frac{p_{1}(\sigma)}{1-P_{1}(\sigma)}\right]^{2}-\frac{p_{1}^{\prime}(\sigma)}{1-P_{1}(\sigma)} \\
\frac{\partial^{3} \ln \left[1-P_{1}(\sigma)\right]}{\partial \sigma^{3}}=-2\left[\frac{p_{1}(\sigma)}{1-P_{1}(\sigma)}\right]^{3}-\frac{3 p_{1}(\sigma) p_{1}^{\prime}(\sigma)}{\left[1-P_{1}(\sigma)\right]^{2}}-\frac{p_{1}^{\prime \prime}(\sigma)}{1-P_{1}(\sigma)}
\end{gathered}
$$

\section{Acknowledgments}

Financial support by the U.S. National Science Foundation under Grant CMS-0556323 to Northwestern University is gratefully acknowledged. The work was started during J.E.'s visiting appointment at Northwestern University, half of which was supported by a Fulbright-Masaryk grant from the Fulbright Foundation and half by the aforementioned grant. Additional financial support was provided by Ministry of Education, Youth and Sports of the Czech Republic under project number ME10030.

\section{References}

Bartle, A., ed. (1985). "Four major dam failures re-examined." Int. Water Power Dam Constr., 37(11), 33-36, 41-46. 
Bažant, Z. P. (2004). "Scaling theory of quaisbrittle structural failure." Proc. Natl. Acad. Sci. U. S. A., 101(37), 13400-13407.

Bažant, Z. P. (2005). Scaling of structural strength, 2nd Ed., Elsevier, London.

Bažant, Z. P., and Chen, E. P. (1997). "Scaling of structural failure." Appl. Mech. Rev., 50(10), 593-627.

Bažant, Z. P., and Jirásek, M. (2002). "Nonlocal integral formulations of plasticity and damage: Survey of progress." J. Eng. Mech., 128(11), $1119-1149$.

Bažant, Z. P., Le, J.-L., and Bazant, M. Z. (2009). "Scaling of strength and lifetime distributions of quasibrittle structures based on atomistic fracture mechanics." Proc. Natl. Acad. Sci. U. S. A., 106(28), 11484-11489.

Bažant, Z. P., Le, J.-L., and Hoover, C. G. (2010). "Nonlocal boundary layer (NBL) model: Overcoming boundary condition problems in strength statistics and fracture analysis of quasibrittle materials." Proc., 7th Int. Conf. Fracture Mechanics of Concrete and Concrete Structures, Jeju, South Korea/Korea Concrete Institute, Seoul, South Korea, 135-143.

Bažant, Z. P., and Novák, D. (2000). "Probabilistic nonlocal theory for quasibrittle fracture initiation and size effect. I. Theory." J. Eng. Mech., 126(2), 166-174.

Bažant, Z. P., and Pang, S.-D. (2006). "Mechanics based statistics of failure risk of quasibrittle structures and size effect on safety factors." Proc. Natl. Acad. Sci. U. S. A., 103(25), 9434-9439.

Bažant, Z. P., and Pang, S.-D. (2007). "Activation energy based extreme value statistics and size effect in brittle and quasibrittle fracture." J. Mech. Phys. Solids, 55(1), 91-134.

Bažant, Z. P., and Pijaudier-Cabot, G. (1988). "Nonlocal continuum damage, localization instability and convergence.” J. Appl. Mech., 55(2), 287-293.

Bažant, Z. P., and Planas, J. (1998). Fracture and size effect in concrete and other quasibrittle materials, CRC Press, Boca Raton, FL and London.

Bažant, Z. P., Vořechovský, M., and Novak, D. (2007). “Asymptotic prediction of energetic-statistical size effect from deterministic finite element solutions." J. Eng. Mech., 133(2), 153-162.

Bažant, Z. P., and Xi, Y. (1991). "Statistical size effect in quasi-brittle structures: II. Nonlocal theory.” J. Eng. Mech., 117(11), 2623-2640.

Borino, G., Failla, B., and Parrinello, F. (2003). "A symmetric nonlocal damage theory." Int. J. Solids Struct., 40(13-14), 3621-3645.

Breysse, D., and Fokwa, D. (1992). "Influence of disorder of the fracture process of mortar." Proc., 1st Int. Conf., Fracture Mech. of Concrete Struct., Z. P. Bazant, ed., Elsevier, London, 536-541.

Carmeliet, J., and de Borst, R. (1995). "Stochastic approaches for damage evolution in standard and non-standard continua." Int. J. Solids Struct., 32(8-9), 1149-1160.

Dimov, I. T. (2008). Monte Carlo methods for applied scientists, World Scientific, London.

dos Santos, C., Strecker, K., Piorino Neto, F., Moreira de Macedo Silva, O., Aparecido Baldacum, S., and Moreira da Silva, C. R. (2003).
"Evaluation of the reliability of $\mathrm{Si}_{3} \mathrm{~N}_{4}-\mathrm{Al}_{2} \mathrm{O}_{3}-\mathrm{CTR}_{2} \mathrm{O}_{3}$ ceramics through Weibull analysis." Mater. Res., 6(4), 463-467.

Duckett, K. (2005). "Risk analysis and the acceptable probability of failure." Struct. Eng., 83(15), 25-26.

Eliáš, J., and Bažant, Z. P. (2011). "Fracturing in concrete via latticeparticle model." Proc. 2nd Int. Conf. Particle-Based MethodsFundamentals and Applications, Barcelona, Spain.

Grassl, P., and Bažant, Z. P. (2009). "Random lattice-particle simulation of statistical size effect in quasi-brittle structures failing at crack initiation." J. Eng. Mech., 135(2), 85-92.

Haldar, A., and Mahadevan, S. (2000). Probability, reliability and statistical methods in engineering Design, Wiley, New York.

Krayani, A., Pijaudier-Cabot, G., and Dufour, F. (2009). "Boundary effect on weight function in nonlocal damage model." Eng. Fract. Mech., 76(14), 2217-2231.

Le, J.-L., and Bažant, Z. P. (2009). "Finite weakest-link model with zero threshold for strength distribution of dental restorative ceramics." Dent. Mater., 25(5), 641-648.

Le, J.-L., Bažant, Z. P., and Bazant, M. Z. (2011). "Unified nano-mechanics based probabilistic theory of quasibrittle and brittle structures: I. Strength, crack growth, lifetime and scaling." J. Mech. Phys. Solids, 59(7), 1291-1321.

Levy, M., and Salvadori, M. (1992). Why buildings fall down? Norton, New York.

Lin, Q., and Labuz, J. (2011). "Process-zone length from image analysis." Proc. 45th U.S. Rock Mechanics/Geomechanics Symp., Paper No. ARMA 11-405.

Lohbauer, U., Petchelt, A., and Greil, P. (2002). "Lifetime prediction of CAD/CAM dental ceramics." J. Biomed. Mater. Res., 63(6), 780-785.

Melchers, R. E. (1987). Structural reliability, analysis \& prediction, Wiley, New York

Munz, D., and Fett, T. (1999). Ceramics: Mechanical properties, failure behavior, materials selection, Springer-Verlag, Berlin.

Nordic Committee for Building Structures (NKB). (1978). "Recommendation for loading and safety regulations for structural design." $N K B$ Report No. 36, Copenhagen, Denmark.

Pang, S.-D., Bažant, Z. P., and Le, J.-L. (2008). "Statistics of strength of ceramics: Finite weakest-link model and necessity of zero threshold." Int. J. Fract., 154, 131-145.

Pattison, K. (1998). "Why did the dam burst?" Invention Tech., 14(1), $22-31$.

Tinschert, J., Zwez, D., Marx, R., and Ausavice, K. J. (2000). "Structural reliability of alumina-, feldspar-, leucite-, mica- and zirconia-based ceramics." Journal of dentistry, 28(7), 529-535.

Tsai, S. W., and Wu, E. M. (1971). "A general theory of strength for anisotropic materials." J. Compos. Mater., 5(1), 58-80.

Vořechovský, M., and Sadílek, V. (2008). "Computational modeling of size effects in concrete specimens under uniaxial tension." Int. J. Fract., 154(1-2), 27-49.

Weibull, W. (1939). "The phenomenon of rupture in solids." Proc., Royal Swedish Inst. Eng. Res. 153, Stockholm, Sweden. 